\title{
Understanding the buffering effect of social media use on anxiety during the COVID-19 pandemic lockdown
}

Yousri Marzouki $^{1 凶}$, Fatimah Salem Aldossari ${ }^{1} \&$ Giuseppe A. Veltri ${ }^{2}$

During the COVID-19 outbreak, lockdown measures have been deployed worldwide. In the wake of these measures, internet and social media use has reached unprecedented peaks. We hypothesize that social media can, in the context of the pandemic, be a placeholder for collective resilient processes modulated by cognitive and emotional components. An online survey $(N=1408)$ using a cross-sectional design was carried out over nine weeks from the beginning of March 2020 to the end of May 2020. The triangulation via SEM statistical modeling, text mining, and sentiment, discriminant, and entropy analyses revealed the granular functional role of social media use in promoting a positive perception towards stressors during the pandemic. This study provides an empirically tested theoretical framework to understand the evolution of buffering mechanisms of social media use as a result of collective resilience. Recommendations on social media use for future lockdown scenarios were provided.

\footnotetext{
${ }^{1}$ Department of Social Sciences, Qatar University, Doha, Qatar. ${ }^{2}$ Department of Sociology and Social Research, University of Trento, Trento, Italy.

凶email: ymarzouki@qu.edu.qa
} 


\section{Introduction}

he ongoing pandemic of the COVID-19 virus, also referred to as the coronavirus pandemic, is the major global health event of 2020. The lengthy absence of a vaccine, alongside a rising death toll and the absence of a globally effective response to the outbreak, renders this pandemic a clear example of an existential risk, as recently defined by Ord (2020). Existential risks, for Ord, are "new kinds of challenges. They require us to coordinate globally and intergenerationally, in ways that go beyond what we have achieved so far. And they require foresight rather than trial and error. Since they allow no second chances, we need to build institutions to ensure that across our entire future we never once fall victim to such a catastrophe."

In order to limit the propagation of the virus, quarantine measures have been adopted by many countries across the world, but not without drawbacks (WHO, 2020). Qui et al. (2020) have explored the various forms of psychological distress associated with the strict quarantine measures applied in China, concluding that protocols have triggered problems such as panic disorder, anxiety, and depression. Brooks et al. (2020) published a comprehensive and informative meta-analysis of the negative psychological impacts of quarantine, noting in particular the prevalence of post-traumatic stress symptoms, confusion, and anger. Their analysis, however, also offered solutions for mitigating these unwanted effects. Notable among their work's recommendations is a call to raise awareness about the altruistic benefits of quarantine, providing the public with a clear and positive rationale for its continued necessity.

During the outbreak, different forms and degrees of lockdown measures were deployed worldwide. As a result of these measures, Internet and social media usage has been observed in unprecedented magnitudes, when compared with the pre-pandemic period (for example, ref. Effenberger et al., 2020; Fischer, 2020). These remarkable levels of usage have had undeniable psychological impacts. Social media can play a crucial, positive role by providing a platform for people to share their opinions and to relay facts about the crisis, but it also provides an outlet for voicing fear about the pandemic. The latter capacity calls for an understanding of the buffering role that social media can play in shaping the resilience of individuals towards traumatic events, existential risks or threats (of which a pandemic serves as a prime example), but this issue has not yet been addressed systematically. Buffering mechanisms are the result of a positive perception towards the stressors which is promoted by resilience (see Catalano et al., 2011). In the context of COVID-19, however, another psychological paradigm has emerged. The fear of contamination and, more acutely, of death has been introduced as the hypothesis of coronavirus anxiety (Fischhoff, 2020). A fear that is associated with a looming threat as perceived by individuals. This threat is a rapidly approaching danger that humans are innately equipped to detect and to respond to this threat's dynamics, whether the source of the stimulus is moving towards or away from an organism (Neuhoff, 1998). In the context of COVID-19, this anxiety arises in response to dynamically moving rather than static, threats. As adaptation mechanisms, which reduce the negative impact of threats, take increased amounts of time to occur as a result of this mobility, emotional responses are ultimately intensified as the looming threats increase (Riskind and Rector, 2018, p. 16). Such a situation yields extreme uncertainty, preventing individuals from organizing a coherent interpretation of otherwise partial and confusing information (Chater, 2020).

In the face of the unknown, this information and decisional overload can lead uncertainty to take over our cognition. In the highly changeable context of the COVID-19 pandemic, these possibilities are exacerbated, leading to a general situation of uncertainty (Yu and Dayan, 2005). In cognitive psychology studies, the difference between perceived knowledge (i.e., what people think they know) and actual knowledge (i.e., what they really know) has been long-established, well before the inception of social media. Typically, studies have evinced correspondence between these two types of knowledge to be very low (e.g., Radecki and Jaccard, 1995). However, in the presence of an existential risk such as the COVID-19 pandemic, and in an era in which social media reigns supreme, the gap between perceived and actual knowledge is liable to increase significantly and ought, accordingly, to be interrogated closely.

According to the looming vulnerability model (LVM), people build knowledge in the form of probability judgments about the proximity of a threat and its perceived dynamic. If they believe that their distance keeps them at a remove from any potentially harmful outcome, then logically their fear and anxiety should be reduced. This self-perceived knowledge about the threat is a key factor to be measured as we seek to understand responses to the COVID-19 pandemic, and it should be considered in combination with the use of social media, as a related but distinct source of knowledge about this threat. We hypothesize that the anxiety of social media users who are facing an unknown risk may evolve in two directions: towards powerlessness (a loss of control over one's environment) or towards resilience. A powerless state of knowledge often provokes counterintuitive rather than spontaneous actions (Geyer, 2001). In other words, the state of powerlessness can be translated psychologically to describe a perception of looming vulnerability; it entails a "subjective perception that one may be defenseless to the dynamism of rapidly growing threats" (Riskind and Rector, 2018, p. 44).

People with high levels of social interaction, who belong to larger and more complex social networks, are generally expected to mobilize a higher level of social and emotional support (Wills and Filer, 2001). With the inception of social media platforms, this same type of support may take hold in the virtual world. As shown by the influential work of Marzouki et al. (2012), the first Arab revolution proved a case in point, as Facebook played a prominent role in generating a sense of collective consciousness among citizens and activists. The authors describe a novel collective response, whereby citizens used Facebook not only to thwart the media blackout in the country, but also to communicate widespread instances of police abuse against protesters.

One key feature in the work of Marzouki and Oullier $(2012,2015)$ is their description of the emergence of a Virtual Collective Consciousness (VCC), generated by the momentum of complex interactions between individuals sharing common goals and driven by a widespread consensus (see also Alperstein, 2019, p. 204). This strengthening of social ties during times of misfortune is a common feature in resilient processes, and can be augmented by the connections afforded by social media. We may note that social media platforms (such as Facebook, in the case of the Arab Spring) play a functional role not just in connecting people but also in enhancing the buffering effect.

The influential model of stress buffering, associated with social support effects (Meng et al., 2017), has many features that can be mapped onto the VCC model. The stress-buffering model proposed by Cohen and Willis (1985) is based on four support functions that can be transposed to any online platform: selfesteem support, informational support (also referred to as the cognitive guidance support), diffuse support based on the sense of belonging with a social group, and instrumental support based on tangible aid provided to affected persons. According to this model, the more a person appraises a situation as a threat, the higher their stress levels rise, and the more the person lacks an appropriate response to the situation. This explains the associated feeling of helplessness and the accompanying lowering of self-esteem. In 
Table 1 The goodness of fit indices for the SEM model with bootstrapping for each week.

\begin{tabular}{llllllll} 
& Week 1 & Week 2 & Week 3 & Week 5 & Week 6 & Week 8 & Week 9 \\
\hline Chi-test & 1.816 & 1.976 & 2.469 & 1.520 & 1.797 & 1.558 \\
DF & 2 & 2 & 2 & 2 & 2 & 2 & 1.787 \\
$p$-value & 0.524 & 0.478 & 0.447 & 0.588 & 0.516 & 0.560 \\
CFI & 0.990 & 0.988 & 0.980 & 0.991 & 0.990 & 0.991 & 0.490 \\
AIC & 823.451 & 831.117 & 833.788 & 827.062 & 827.671 & 830.808 \\
BIC & 842.305 & 849.971 & 852.642 & 845.916 & 846.525 & 849.661 \\
RMSEA & 0.031 & 0.039 & 0.051 & 0.027 & 0.034 & 0.023 \\
\hline
\end{tabular}

such situations, the benefits offered by social support interventions are twofold: they prevent or mitigate the appraisal of a situation as a threat, and, when full prevention of such an appraisal is not possible, they make available effective coping strategies.

In the case of the COVID-19 pandemic, the situation is almost unavoidably appraised as stressful since, as we have already argued, the global scale of the event renders it an existential risk. The nature of the pandemic and the various lockdowns imposed in turn, furthermore, mean that the majority of social support, like other forms of communication, has so far been offered through online and social media platforms. The LVM model stipulates that anxiety can be the result of an overestimation of the probability or proximity of threats. We believe this can explain fear towards COVID-19, in the absence of buffering mechanisms likely to be provided by social media. We hypothesize that social media can be a placeholder for collective resilient processes modulated by cognitive and emotional components during a pandemic.

\section{Methods}

We have conducted an online survey study using a cross-sectional survey design (see Lavrakas, 2008). Since the study was conducted amid a global lockdown period, the optimal strategy was a snowball sampling technique that was implemented by persistently inviting students, colleagues, and acquaintances to share the link to the online survey in their respective networks. All participants were anonymous Internet users voluntarily completing the questionnaire by simply following the link posted on their social media networks.

A total of 1408 answers were collected from the beginning of the study in March 2020 (timestamp: 3/20/2020 2:51:58 p.m.) to its end in May 2020 (timestamp: 5/21/2020 10:12:29 p.m.). Nine cross-sections points were defined weekly. The collected answers for each week are distributed as follows: week 1 (from 3/20/2020 2:51:58 p.m. to 3/27/2020 7:58:49 p.m., $n=477$ ), week 2 (from 3/ 28/2020 7:46:18 p.m. to 4/3/2020 11:49:23 p.m., $n=62$ ), week 3 (from 4/4/2020 12:19:15 a.m. to 4/9/2020 4:34:04 p.m., $n=141$ ), week 4 (from 4/10/2020 12:45:32 p.m. to 4/12/2020 2:28:18 a.m., $n=4$ ), week 5 (from 4/17/2020 6:37:30 p.m. to 4/24/2020 1:58:09 p.m., $n=88$ ), week 6 (from 4/25/2020 10:01:46 a.m. to 5/2/2020 7:52:37 p.m., $n=369$ ), week 7 (from 5/3/2020 12:00:11 a.m. to 5/ 8/2020 4:39:11 a.m., $n=72$ ), week 8 (from 5/12/2020 10:59:12 p. m. to 5/18/2020 11:46:48 a.m., $n=117$ ), and week 9 (from 5/19/ 2020 3:22:19 a.m. to 5/21/2020 10:12:29 p.m., $n=78$ ). Only week 4 was dropped from the analysis, given the very small number of recorded answers $(n=4) .9 .16 \%$ of participants did not provide verbatim responses (Lavrakas, 2008). Among the 1279 verbatim responses, $70.21 \%$ were in the Arabic language, 22.36\% in English, and $7.43 \%$ in French.

\section{Results}

The structural relationship analysis. The measurement part of the SEM is to validate the cognitive dimensions across the three exogenous variables. The structural part of the SEM is to examine the impact of perceived knowledge on the fear of coronavirus. We have tested this relationship through a structural equation model where the three observed exogenous variables are supposed to be reflected by a latent variable that captures the following three dimensions of perception: social media use for social knowledge about the pandemic, self-perceived knowledge for the subjective knowledge of the users about the pandemic, and the perception of threat for the knowledge associated with death saliency and the estimation of the probability of death. The endogenous variable is Anxiety (i.e., fear).

We tested the same model for each week during the 9 weeks of the study (except for weeks 4 and 7 where the lack of responses hinders the SEM model). Preliminary data screening did not reveal missing data, since individual responses to the survey could only be recorded if the participant responded to all sections. Full completion of the survey was mandatory, in short, before responses could be validated. Table 1 shows the fit indices of the conceived model for each week after running SEM modeling using the $R$ package lavaan (Rosseel, 2012) with 50 draws of bootstrapping. By assuming that the data collected are continuous, the estimation of the model parameters used maximum likelihood.

Figure 1 shows that the evolution across time of the structural relationship between the latent construct (i.e., perceived knowledge) and the relative contribution to the endogenous variable (i.e., fear) is consistent, while exhibiting some variations as the study progressed. The Comparative Fit Index (CFI) for each model is higher than the cut-off value of .90 for the best fit, and the Root Mean Square Error of Approximation (RMSEA) is lower for each model than the cut-off value of 0.08 for a good fit. These goodness-of-fit indices suggest that the fit of the data to the hypothesized model was adequate.

It is worth highlighting the trade-off pattern between selfknowledge and threat perception, given the weight of social media use on the latent perceived knowledge variable. Overall, the more Self-knowledge decreases in impact, the more Threat perception gains in impact, and vice versa, while the weight of Social media use is conditioning the weight of the latent variable on Fear.

A deeper exploration of the relationship between the observed exogenous variables and the latent factor is presented together with a later account of the content of the cognition, analyzed through verbatim data.

Text corpus analysis. Participants in our study were asked to provide up to five words that they associated with their conditions while completing the questionnaire. These five keywords for each participant were analyzed as a textual corpus $(N=862)$, upon which we performed cluster analysis and psycholinguistic classification.

Cluster analysis. In the case of unsupervised clustering of the corpus, the analysis procedure consists of the following steps:

1. Construction of a data table context units $\times$ lexical units (up to 300,000 rows $\times 3000$ columns), with presence/absence values; 

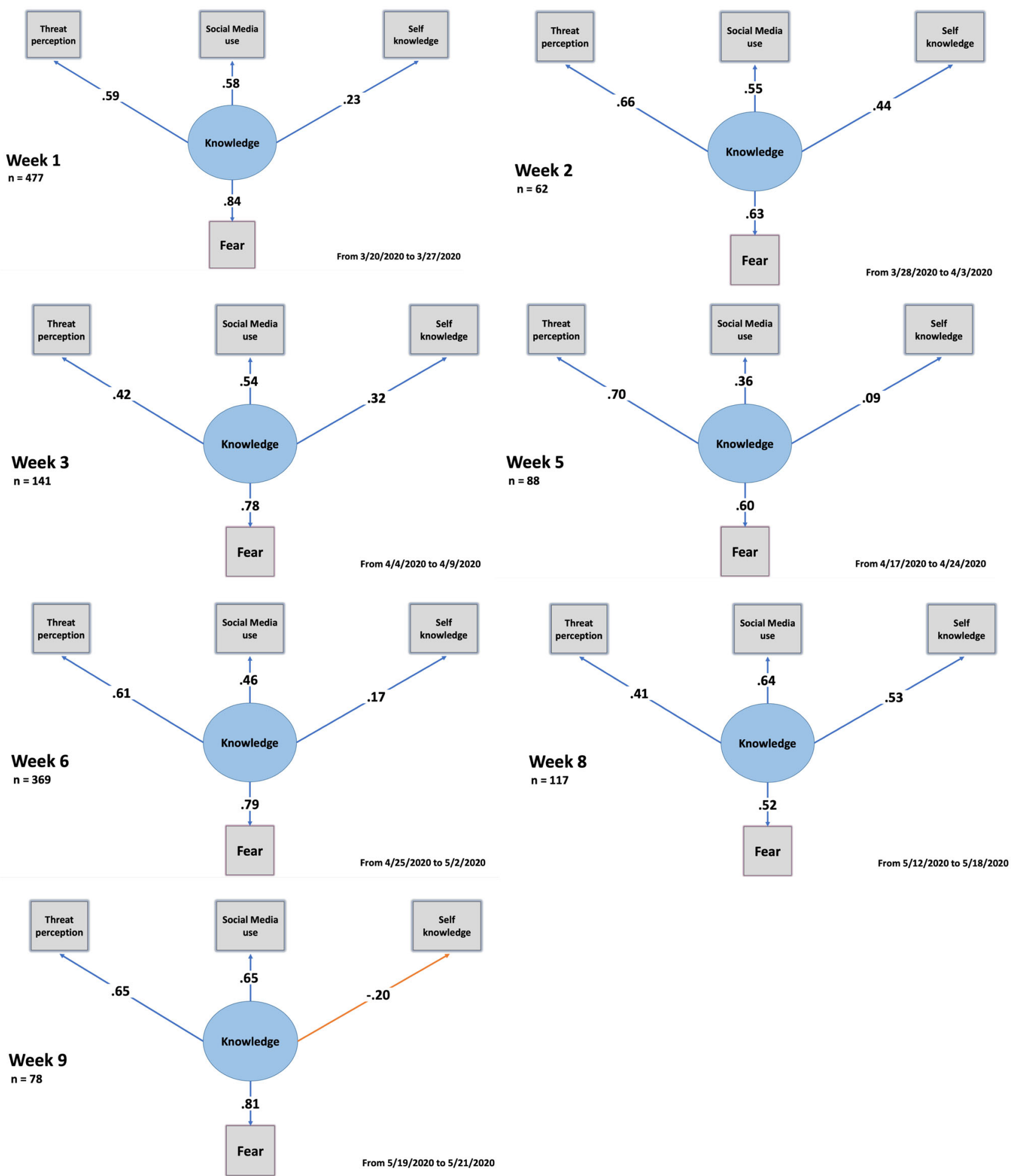

Fig. 1 Illustration of the Structural Equation Model. SEM model with standardized structural coefficient estimates of the relationship between the independent variables, the latent variable "perceived knowledge", and Fear in each week.

2. TF-IDF normalization and scaling of row vectors to unit length (Euclidean norm);

3. Clustering of the context units (measures: cosine coefficient; method: bisecting K-means (see Steinbach et al., 2000; Savaresi and Boley, 2004; Savaresi and Boley, 2011).

4. Filing of the obtained partitions and, for each of them:

a. Construction of a contingency table lexical units $\times$ clusters $(n \times k)$; b. Computing the chi-square test applied to all the intersections of the contingency table;

c. Performing the correspondence analysis of the contingency table lexical units $\times$ clusters (see Benzécri and Benzécri, 1984; Greenacre, 1984; Lebart and Salem, 1994).

The unsupervised clustering of the context units (see step ' 3 ' above) can be performed either by using the bisecting K-means algorithm (1) or by using a non-centered version of PDDP (i.e., 


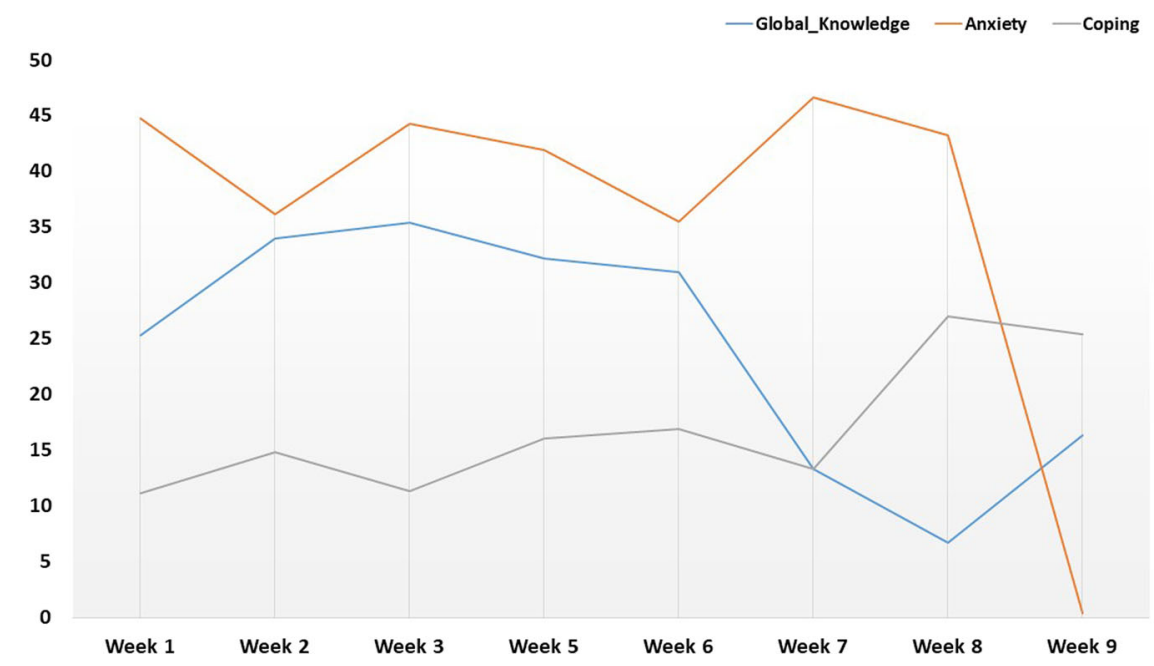

Fig. 2 The clusters' unique use. Evolution of the per cent of the unique use of the three relevant clusters over weeks.

Principal Direction Divisive Partitioning, Boley, 1998) for selecting the seeds of each K-means bisection. The main differences between the above methods depends on how the two seeds of each bisection are computed; in fact, in the (1) case they result from an iterative procedure whereas in the (2) case they are computed through SVD (i.e., Singular Value Decomposition), i.e., through a 'one-shot' algorithm (see Savaresi and Boley, 2011). We opted for a K-means bisection algorithm.

The findings of the text corpus analysis, in terms of clusters between co-occurring words, showed the presence of four main clusters that we labeled as follows: Cluster 1. Global knowledge, Cluster 2. Anxiety, Cluster 3. Symptoms, Cluster 4. Coping. Figure 2 reveals the distribution of the relative presence of the three relevant clusters for this study: 'Global Knowledge', 'Anxiety', and 'Coping'. These labels are assigned considering the words with the highest chi-square values in each cluster.

Further visualization of the corpus analysis includes:

Co-occurrence matrix. A co-occurrence matrix between the keywords (bi-grams) out of the corpus with a minimum.

frequency of four $(N=165)$ was generated to visualize a potential thematic clustering (see Fig. 3). Figure 3 shows the cooccurrence matrix of the most frequent keywords (bi-grams) in the verbatim data. Clusters of co-occurring words are a first indication of potential emergent themes ${ }^{1}$.

$3 D$ modeling of the proximity between clusters. Discriminant analysis of thematic clusters as a function of the observed study variables $^{2}$

Next, we performed a discriminant function analysis for each week's data to determine if the aggregate levels of fear, social media usage, self-knowledge about COVID-19, and perceived threat, discriminated between the clusters obtained from the verbatim data and assigned to each participant as a categoricalgroup variable (as the dominant theme of his or her self-reported text). The results revealed that the most significant results for the discriminant function of the predictors were observed in weeks 6 , 7, and 8 (Fig. 4). During this period, all covariates contributed significantly to discriminating between the four clusters (see Table S1, Supplementary Information for more details). This can be interpreted as indicating that, in the above-mentioned weeks, the thematic clusters were associated with different combinations of the four relevant variables: fear, social media usage, self-knowledge, and threat.
Sentiment analysis. LIWC (linguistic inquiry and word count) is a specialized software for studying language use and its association with the psychological, cognitive, and emotional states of the text producer. In classifying and analyzing the corpus using LIWC (Pennebaker et al., 2007), it is possible to calculate the extent to which various 'categories' of word are present in the corpus. The LIWC program encompasses a main text analysis module along with a group of built-in dictionaries that allow for the quick classification of a corpus. For example, we might discover that $5.67 \%$ of the words in a given body of text are about family and $3.38 \%$ are about sadness. The LIWC output lists all LIWC categories and shows how much each category has featured in a given text. Dictionaries are central in the LIWC software because a dictionary refers to the collection of words that define a particular category. The dictionaries in LIWC2015 have gone through a long period of testing, resulting in the current version (Pennebaker et al., 2007). Most relevant for our concerns, the 80 language categories in LIWC have been linked in a large body of studies to significant psychological processes. LIWC categories span sentiment but also other cognitive categories such as space, time, modes of cognitive processing, and social domain.

According to the VCC model, the ability to keep working toward a goal in the face of difficulties can be enhanced by online interactions. This makes social networking a significant outlet for resilience, buttressed by the presence of positive feedback and commonly shared values held by other users. This assumption can be tested by tracing the valence (the positive and negative dimensions) of words appearing in each week's responses. The percentage of words associated with risk was, across the study, highest, followed by words about social ties (family, friends, and social connections). The results showed a cyclic pattern with a clear turning point in week 5 , which demonstrated a peak in the use of words related to cognitive processing and a decrease in words loaded with negative emotions (see Fig. 5). Moreover, the progressive increase of positively-valenced words coincided with a decrease in the use of words related to risk.

Overall, the decrease in the use of emotionally loaded words denotes the presence of a shift in the network's shared knowledge and collective narrative. The initial narrative, mainly marked by emotional content, reflects the absence of adjustive counterresponses to the threat, of the kind we might expect to be provided by the stress-buffering of social support effects. However, the increase of cognitive words in the new narrative, taking effect from week five, reflects the presence of a newly effective appraisal process according to the same model. 


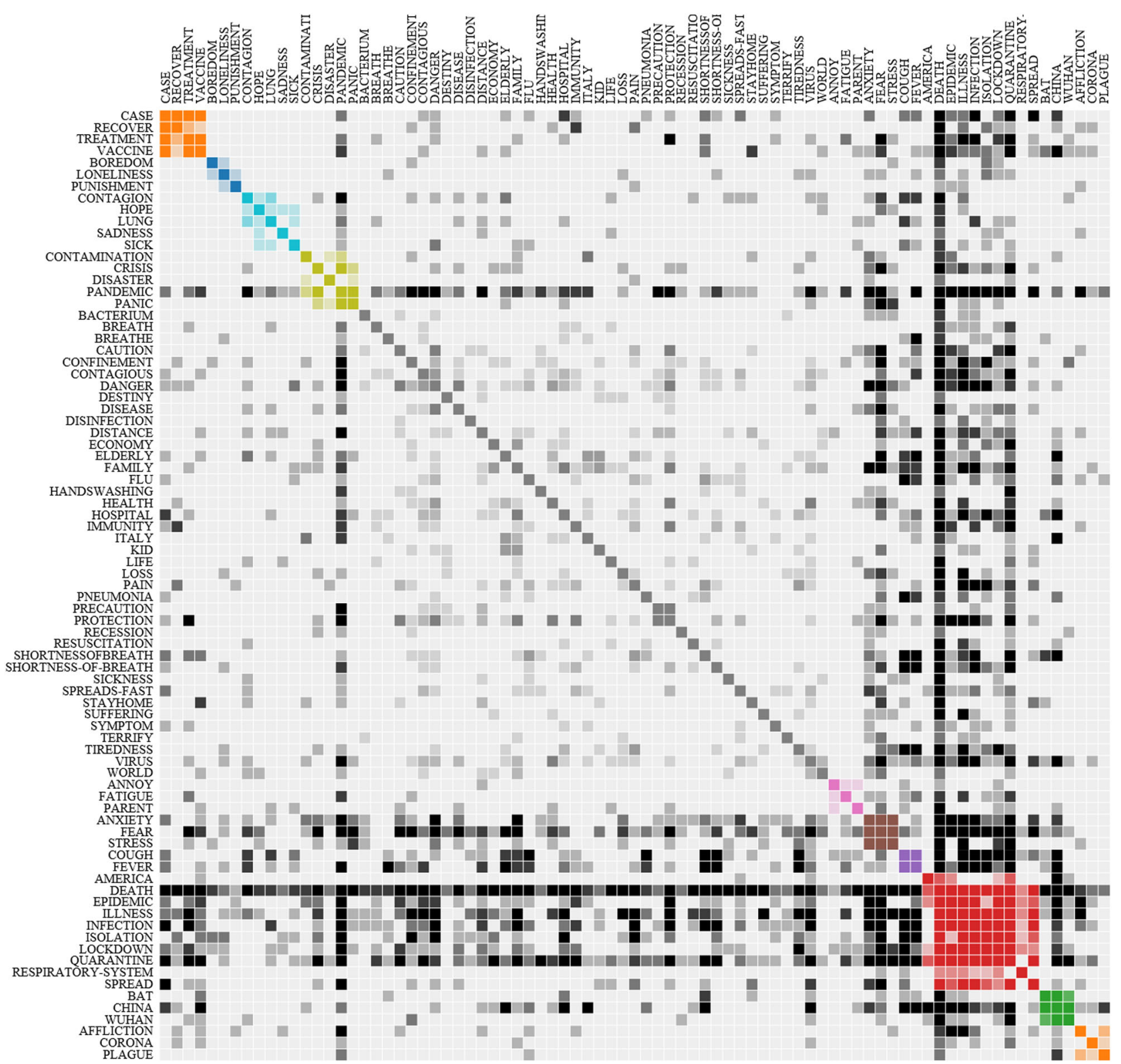

Fig. 3 The co-occurence matrix. Graphical representation of within n-grams word co-occurrences matrix (specifically bi-grams). Different colors indicate clusters; Color shades stand for weights; Values are word co-occurrences.

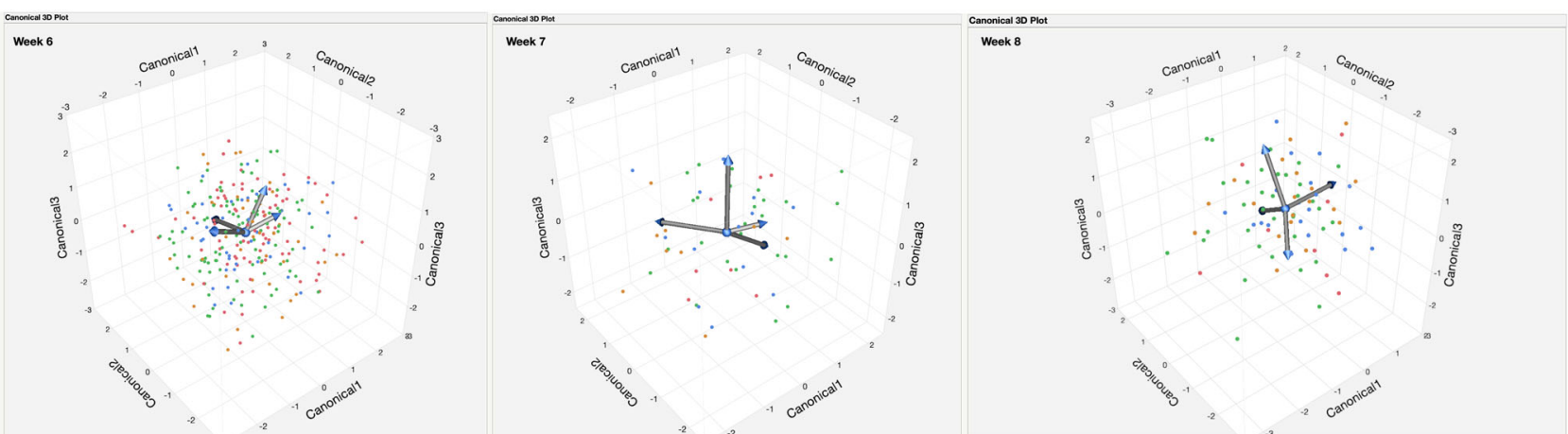

Fig. 4 3D canonical Plot for the linear discriminant analysis. The points have been colored by clusters. These define the three dimensions that provide maximum separation among the groups. Each canonical variable is a linear combination of the covariates. 

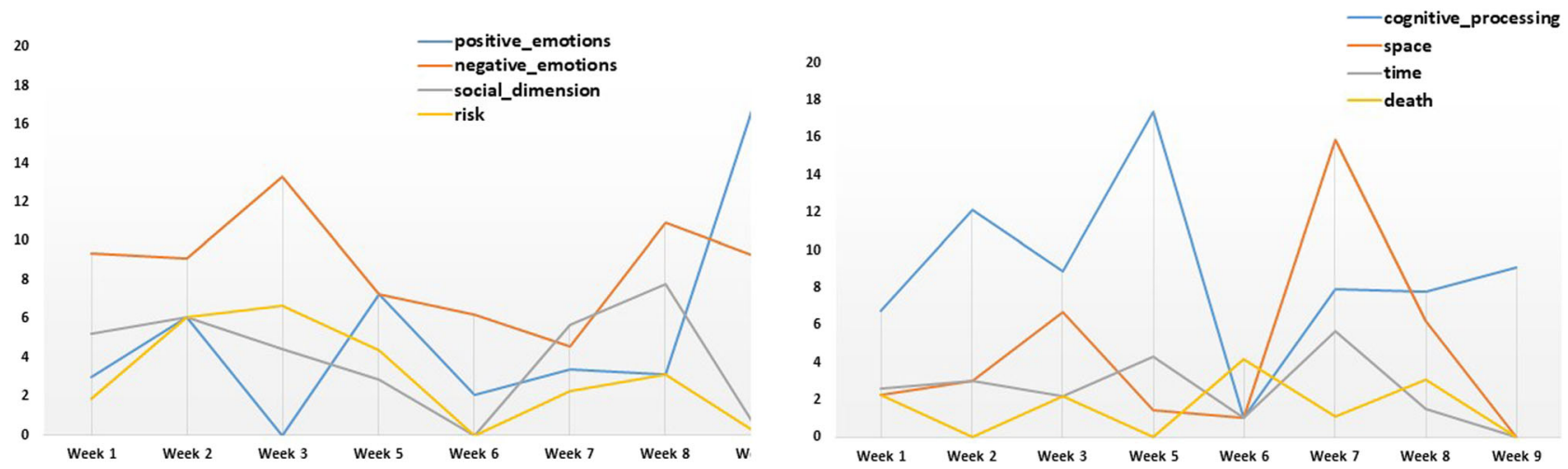

Fig. 5 Sentiment and cognitive categories of the words. Evolution of the per cent of words associated with valence, social dimension, and risk over the weeks in the left panel; and with cognitive processing, space, time, and death in the right panel.

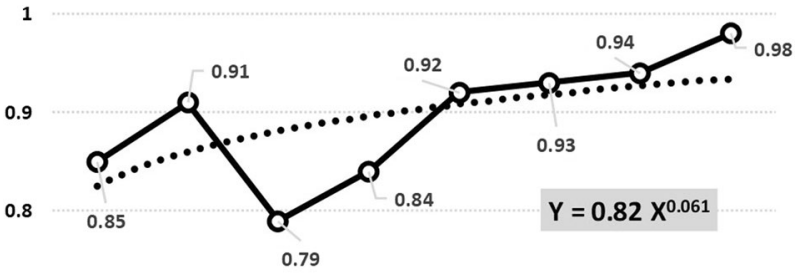

0.7

0.6

0.5

week1 week2 week3 week5 week6 week7 week8 week9

Fig. 6 Entropy evolution. Normed entropy based on the cluster membership probability value for each word as a function of weeks.

Entropy analysis. The measure of corpus diversity is a key information that may reflect the underlying mindset of a collective verbatim content and how it can evolves over time (Shaikh et al., 2017; Feldman et al., 2018; Marzouki et al., 2020). In this regard, we have calculated entropy as a metric of information uncertainty that also reflects social diversity (Shannon and Weaver, 1975). We have computed the entropy index based on the simple social entropy algorithm advocated by Balch (2000). Indeed, we assumed that each word emanated from an agent (i.e., social network user), and that each word belongs to a category (i.e., semantic cluster) based on the cluster membership probability value. Figure 6 shows the normed entropy (minimum value is 0 and the maximum is 1) as a function of weeks. When the entropy is at its maximum value, the content is likely to fall into different dimensions. When the entropy is minimized, the content is likely to fall into one possible dimension. The results showed for the first 5 weeks a normed entropy average equal to 0.85 and an average of 0.94 for the remaining 4 weeks with a trend tending towards its asymptote. Thus, in the presence of a high incidence of loaded words associated with negative emotions, the corpus tends to be more coherent and indicative of some sort of consensus between users. However, the shift started with the sharp occurrence of words related to cognitive processes, which are more likely to trigger diversity according to this entropy analysis.

\section{Discussion}

This paper provides an understanding of the buffering role played by social media use as individuals and communities coped (and continue to cope) with the COVID-19 pandemic. To this end, we have collected data through an online cross-sectional survey to analyze the structural relationship between social media usage and several aspects of coping with the pandemic.

Triangulation via four main results stemming from different analysis techniques showcases a compelling empirical case for the granular functional role of social media use, a role that remains highly flexible and dynamic. The trade-off between selfknowledge and threat perception is systematically impacted by social media use. This trade-off effect is also evidenced by the way the evolution curves of the 'coping' and 'anxiety' semantic clusters mirror each other closely.

Sentiment analysis supports the same claim when two competing dimensions, the emotional component and the cognitive one, are measured; when the latter rises. the former falls. Again, the findings pinpoint the period around week 4 and 5 as a clear turning point, a transitional state of the users' self-perceived knowledge.

In other words, the perception by many social agents of a particular reality in a particular period leans towards a consensus (i.e., less diversity) when they are exposed to a negatively loaded event (in this case, a pandemic). The briefly formed virtual collective consciousness, as shown in the tentative model presented in Fig. 7, feeds back individual perceptions with expectations, leading to the consolidation of fear and anxiety as a major response. However, a cognitive appraisal of this emotion can also be posited and is mirrored by the presence of coping mechanisms through social norms, rules of thumb, heuristics, etc. (see Cialdini, 2009). These ultimately act to reduce the original fear: educated opinions are exchanged and individuals adapt purposefully to shared online content, thus fighting the contagion of fear even as they face the unknown.

The discrepancy between self-perceived knowledge and actual knowledge of COVID-19 has been the subject of a recent scientific investigation (Granderath et al., 2020). The findings indicate that individuals who felt more threatened by COVID-19 were more likely to seek information about it. The results also reveal a positive, if complex, correlation between the volume of media encountered and knowledge: as individuals encounter a higher volume of media, their self-perceived knowledge increases but not their actual knowledge. Our findings extend these results to the case of social media.

In line with the uncertainty reduction theory (Berger, 1986), the pandemic has generated a considerable amount of uncertainty that has triggered a widespread information-seeking process (Burke et al., 2010). Information has been sought largely through social media networks, which have served as the primary media outlet for most individuals during lockdown periods. Information-seeking tends to progressively reduce uncertainty, as converging ideas and 


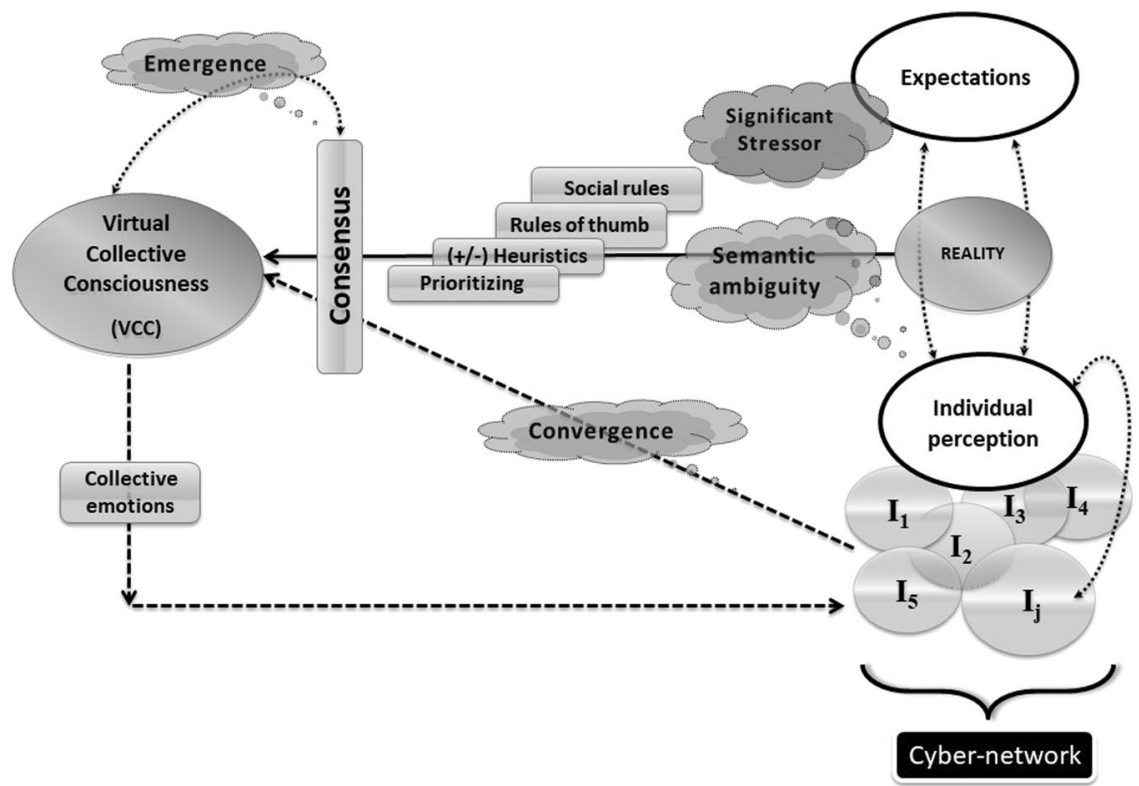

Fig. 7 The VCC dynamic model. A tentative model to account for the dynamics of users' knowledge via the interaction with the cybernetwork and the perceived virtual collective consciousness when facing a significant stressor.

feelings towards the source of the threat are perceived within the user's network. The semantic ambiguity presented in Fig. 7 matches the initial stance of uncertainty, which is increased by a lack of consensus among users. The cognitive appraisal of the threat (i.e., the pandemic) following information seeking involves a number of mechanism such as social rules, rule of thumbs, positive vs. negative heuristics, and prioritizing that once shared by different users will help reduce the divergence and promotes consensus.

In the vein of Marzouki et al. (2020), we interpret decreasing entropy as an indicator of collectively shared reactions, opinions, and attitudes towards COVID-19 (these converging responses are referred to as a consensus in Fig. 7). The results of the entropy analysis revealed a clear increase over time in lexical diversity, aligned with an increase of words semantically related to cognitive processes. Indeed, the users' convergence in expressing their perception through negative words was particularly salient in the first period and ultimately led to a collective coherent perception based on shared knowledge revolving around negative thoughts towards the pandemic. The shift after five weeks, as shown by the results, denotes the clear emergence of a reappraisal of the negative situation through social support originating from social media platforms. According to the model proposed by Cohen and Willis (1985), which emphasizes the stress-buffering of social support effects, this shift can be interpreted as a "collective adjustive counter-response".

\section{Limitations}

Two limitations of our study that can be mentioned are the fact that we do not have a probabilistic sample, and the fact that the exact estimation of the incidence of the indicators is not determined. Instead, this study explores the structural relationship between these constructs, which were directly drawn from the Looming Vulnerability Model. By design, moreover, we did not choose to collect socio-demographic variables such as education level, gender, digital literacy, and socio-economic status, which could be relevant as covariates in the SEM models. This choice purposefully aimed to keep the data collection with a very small cognitive load footprint for the participants who completed the questionnaire during the lockdown phase.

\section{Recommendations}

According to a UNICEF statement (Fore, 2020) published on 9 April 2020, 82 countries applied lockdown measures, including $7 \%$ with a full and $53 \%$ with a partial form of lockdown, in a period where the number of COVID-19 cases worldwide was nearly 2 million. As of 24 December 2020, the worldwide number of confirmed cases is surpassing 79 million across 188 countries, according to the COVID-19 dashboard ${ }^{3}$ created by Johns Hopkins University's Whiting School of Engineering. Kissler et al. (2020) have projected the epidemic dynamics of COVID-19 over the next five years, and their deterministic modeling approach reveals that an acute resurgence in COVID-19 is more likely to happen in the absence of interventions. However the virus develops, prolonged or intermittent lockdowns will probably remain necessary, given the likelihood of heavy pressure on health care systems, particularly during concomitant seasonal peaks of other diseases, mainly influenza.

A recent commentary by Habersaat et al. (2020) proposed ten considerations to manage the COVID-19 crisis, which included recommendations for fostering resilience in communities. Given the extent of lockdown measures, online communities are the spaces where such forms of resilience may emerge and take hold. Indeed, from a psychological perspective, our study reveals that social media use plays the crucial role of a buffer, insofar as it manages and mitigates the forms of anxiety experienced by our respondents during the pandemic. Therefore, social media should play a pivotal role in all projected scenarios. As we plan for future, successive phases of lockdown and seek to mitigate their negative psychological effects, we should boldly harness social media's capacities of promoting resilience and facilitating coping strategies.

Since the widespread adoption of social networking, new and rapidly changing modes of communication are part of the lives of humans across the world. Online social networks are now essential tools to build and raise awareness about many social issues. Existential risks, such as the COVID-19 pandemic, are assumed to be unprecedented situations that require specific types of psychological responses. The merit of this study is to shed light on how these responses can be facilitated and improved through the use of social media. Moreover, the current findings will eventually pave the way 
for further research and policies, as we acquire a better understanding of how to confront similar risks and threats in the future. A few key elements of prevention and intervention can be highlighted as guidelines for public health communication strategies:

1. Reduce uncertainty about the threat, and allow people to reframe their uncertainty in a positive way, by promoting constantly trustworthy sources of information.

2. Heed the importance of a cyclic pattern inherent to human emotional reactions in order to adjust the content of shared online material and to speed up the establishment of collective resilience.

3. Use online platforms to cement and embrace social norms that emphasize the promotion of protective behaviors.

4. Boost the buffering effect of social media by guiding users to socialize online in an effective and organized way during lockdown phases, through scheduling daily social online activities with family, friends, colleagues, and broader social actors.

Areas for future research, stemming from this study, include the question of how social media use has mediating or interacting effects with other independent variables that determine how individuals cope with threats. This study could also be complemented by a comparative approach, which would address how differences between social networking platforms (Facebook, YouTube, and Twitter, for example) may help us to understand the subtleties of the structural relationship between threats and resilience. Finally, collective resilience processes are not exempt from cultural impacts (see Neher and Miola, 2016). Breaking down our analysis by geographical areas, therefore, could be highly informative as we seek to understand how online collective coping strategies are mediated.

\section{Data availability}

The datasets generated during and/or analyzed during this study are available in an Open Science Framework repository directory. Links to datasets are as follows: The raw data: https://mfr.de-1.osf. io/render?url=https://osf.io/saz92/?direct $\% 26$ mode $=$ render $\%$ 26 action $=$ download $\% 26$ mode $=$ render. The merged data with clusters' variables: https://mfr.de-1.osf.io/render?url=https://osf. io/bw4r3/?direct $\% 26$ mode $=$ render $\% 26$ action=download $\%$ 26 mode $=$ render. Raw data for text corpus analysis: https://mfr. de-1.osf.io/render?url=https://osf.io/8auvx/?direct\% 26 mode $=$ render $\% 26$ action $=$ download $\% 26$ mode $=$ render.

Received: 6 July 2020; Accepted: 13 January 2021;

Published online: 15 February 2021

\section{Notes}

1 The link to an HTML animated version of Fig. 3 can be downloaded via the following link https://mfr.de-1.osf.io/render?url=https://osf.io/yc9te/?direct $\% 26$ mode $=$ render $\%$ 26action $=$ download $\% 26$ mode $=$ render.

2 The following link represents a 3D animated video of a dynamic graph of the clusters in the space detected by the correspondence analysis: https://www.youtube.com/ watch? $\mathrm{v}=\mathrm{jAGw} 1 \mathrm{X} 6 \mathrm{sJPU}$. It is a visualization of thematic clusters and their relationships using a $3 \mathrm{D}$ moving animation.

3 The link to the Dashboard: https://www.arcgis.com/apps/opsdashboard/index.html\#/ bda7594740fd40299423467b48e9ecf6.

\section{References}

Alperstein NM (2019) Social movements: our virtual collective consciousness. In: Alperstein NM (ed) Celebrity and mediated social connections. Springer International Publishing, p. 195

Balch T (2000) Hierarchic social entropy: an information theoretic measure of robot group diversity. Autonom Robots 8:209-238
Benzécri JP, Benzécri F (1984) Pratique de l’analyse des données Tome 1: analyse des correspondances, exposé élémentaire. Dunod, Paris

Berger CR (1986) Uncertain outcome values in predicted relationships: uncertainty reduction theory then and now. Hum Com Res 13:34-38

Boley D (1998) Principal direction divisive partitioning. Data Mining Knowl Discov 2:325-344. https://doi.org/10.1023/A:1009740529316

Brooks SK, Webster RK, Smith LE, Woodland L, Wessely S, Greenberg N, Rubin GJ et al. (2020) The psychological impact of quarantine and how to reduce it: a rapid review of the evidence Lancet 395:912-920. https://doi.org/10.1016/ S0140-6736(20)30460-8

Burke JA, Spence PR, Lachlan KA (2010) Crisis preparation, media use, and information seeking during Hurricane Ike: lessons learned for emergency communication. J Emergency Manag 8:27. https://doi.org/10.5055/jem.2010.0030

Catalano D, Chan F, Wilson L, Chiu CY, Muller VR (2011) The buffering effect of resilience on depression among individuals with spinal cord injury: a structural equation model. Reh Psych 56:200-211. https://doi.org/10.1037/a0024571

Chater N (2020) Facing up to the uncertainties of COVID-19. Nat Hum Behav 4:439-439. https://doi.org/10.1038/s41562-020-0865-2

Cialdini RB (2009) Influence: the psychology of persuasion. Harper Collins eBooks Cohen S, Wills TA (1985) Stress, social support, and the buffering hypothesis Psych Bul 98:310-357

Effenberger M, Kronbichler A, Shin JI, Mayer G, Tilg H, Perco P et al. (2020) Association of the COVID-19 pandemic with Internet search volumes: a google trends TM analysis Int J Infect Dis 95:192-197. https://doi.org/ 10.1016/j.ijid.2020.04.033

Feldman LB, Aragon CR, Chen NC, Kroll JF (2018) Emoticons in informal text communication: a new window on bilingual alignment. Bilingualism 21:209-218. https://doi.org/10.1017/S1366728917000359

Fischer S (2020) Social media use spikes during the pandemic. AXIOS. https:// www.axios.com/social-media-overuse-spikes-in-coronavirus-pandemic764b384d-a0ee-4787-bd19-7e7297f6d6ec.html. Accessed 10 May 2020

Fischhoff B (2020) Speaking of Psychology: Coronavirus Anxiety (Part 1). https:// www.apa.org/research/action/speaking-of-psychology/coronavirus-anxiety. Accessed 9 Apr 2020

Fore H. (2020) Don't let children be the hidden victims of COVID-19 pandemic. https://www.unicef.org/press-releases/dont-let-children-be-hidden-victimscovid-19-pandemic Accessed 20 Apr 2020

Geyer F (2001) Alienation, Sociology of. In: Smelser NJ, Baltes PB (eds) International encyclopedia of the social and behavioral sciences. Pergamon Press, pp. 388-392

Granderath JS, Sondermann C, Martin A, Merkt M (2020) The effect of information behavior in media on perceived and actual knowledge about the COVID-19 pandemic. https://psyarxiv.com/3y874/

Greenacre MJ (1984) Theory and applications of correspondence analysis. Academic Press, London

Habersaat KB, Betsch C, Danchin M, Sunstein CR, Böhm R, Falk A, Brewer NT Omer SB, Scherzer M, Sah S, Fischer EF (2020) Ten considerations for effectively managing the COVID-19 transition. Nat Hum Behav https://doi. org/10.1038/s41562-020-0906-x

Kissler SM, Tedijanto C, Goldstein E, Grad YH, Lipsitch M (2020) Projecting the transmission dynamics of SARS-CoV-2 through the postpandemic period. Science 368:860-868. https://doi.org/10.1126/science.abb5793

Lavrakas P (2008) Encyclopedia of survey research methods. Sage Publications, Inc. https://doi.org/10.4135/9781412963947

Lebart L, Salem A (1994) Statistique textuelle. Dunod, Paris

Ord T (2020) The precipice: existential risk and the future of humanity. [E-reader version]. Bloomsbury, p. 18

Marzouki Y, Barach E, Srinivasan V, Shaikh S, Feldman LB (2020) The dynamics of negative stereotypes as revealed by tweeting behavior in the aftermath of the Charlie Hebdo terrorist attack. Heliyon 6:e04311. https://doi.org/10.1016/j. heliyon.2020.e 04311

Marzouki Y, Oullier O (2015) Internet search volume as a proxy approach to the Virtual Collective Consciousness. In: Cheok, AD (ed) Hyperconnectivity and the future of Internet communication, LAP LAMBERT Academic Publishing

Marzouki Y, Oullier O (2012) Revolutionizing revolutions: Virtual Collective Consciousness and the Arab Spring. THE HUFFINGTON POST. https:// www.huffpost.com/entry/revolutionizing-revolutio_b_1679181. Accessed 23 Feb 2018

Marzouki Y, Skandrani-Marzouki I, Béjaoui M, Hammoudi H, Bellaj T (2012) The Contribution of Facebook to the 2011 Tunisian revolution: a cyberpsychological insight. Cyberpsy Beh Soc Net 15:237-244. https://doi.org/10.1089/ cyber.2011.0177

Meng J, Martinez L, Holmstrom A, Chung M, Cox J (2017) Research on Social Networking Sites and Social Support from 2004 to 2015: A Narrative Review and Directions for Future Research. Cyberpsy Beh Soc Net 20:44-51. https:// doi.org/10.1089/cyber.2016.0325

Neher F, Miola A (2016) European Commission and Joint Research Centre. Culture and resilience. Available via https://publications.jrc.ec.europa.eu/ repository/bitstream/JRC103748/lb-na-28314-en-n\%20.pdf 
Neuhoff JG (1998) Perceptual bias for rising tones. Nature 395:123-124. https:// doi.org/10.1038/25862

Pennebaker JW, Chung C, Ireland M, Gonzales M, Booth RJ (2007) The development and psychometric properties of LIWC2007. https://www.liwc.net/ LIWC2007LanguageManual.pdf

Qiu J, Shen B, Zhao M, Wang Z, Xie B, Xu Y et al. (2020) A nationwide survey of psychological distress among Chinese people in the COVID-19 epidemic: implications and policy recommendations Gen Psych 33:e100213. https://doi. org/10.1136/gpsych-2020-100213

Radecki CM, Jaccard J (1995) Perceptions of knowledge, actual knowledge, and information search behavior. J Exp Soc Psych 31:107-138. https://doi.org/ 10.1006/jesp.1995.1006

Riskind JH, Rector NA (2018) Looming vulnerability: theory, research and practice in anxiety. Springer, New York

Rosseel Y (2012) lavaan: an R package for structural equation modeling. J Stat Soft 48, https://doi.org/10.18637/jss.v048.i02

Savaresi SM, Boley D (2011) On the performance of bisecting K-means and PDDP. In: V Kumar and R Grossman (eds) Proceedings of the First SIAM International Conference on Data Mining. SIAM, Chicago

Savaresi SM, Boley DA (2004) Comparative analysis on the bisecting K-means and the PDDP clustering algorithms. Intel Data Analysis 8:345-362

Shaikh S, Feldman LB, Barach E, Marzouki Y (2017) Tweet sentiment analysis with pronoun choice reveals online community dynamics in response to crisis events. In: Schatz S, Hoffman M (eds) Advances in Cross-Cultural Decision Making, vol. 480. Springer, International Publishing, pp. 345-356

Shannon CE, Weaver W (1975) The mathematical theory of communication. University of Illinois Press

Steinbach M, Karypis G, Kumar VA (2000) Comparison of document clustering techniques. proceedings of world text mining conference. KDD, Boston

WHO (2020) Considerations for quarantine of individuals in the context of containment for coronavirus disease (COVID-19). https://apps.who.int/iris/ handle/10665/331497 Accessed 24 May 2020

Wills TA, Filer M (2001) Social networks and social support. In: Baum A, Revenson TA, Singer JE (eds) Handbook of health psychology. Psychology Press, pp. 209-234
Yu AJ, Dayan P (2005) Uncertainty, neuromodulation, and attention. Neuron 46:681-692. https://doi.org/10.1016/j.neuron.2005.04.026

\section{Funding}

Open access funding provided by the Qatar National Library.

\section{Competing interests}

The authors declare no competing interests.

\section{Additional information}

Supplementary information The online version contains supplementary material available at https://doi.org/10.1057/s41599-021-00724-x.

Correspondence and requests for materials should be addressed to Y.M.

Reprints and permission information is available at http://www.nature.com/reprints

Publisher's note Springer Nature remains neutral with regard to jurisdictional claims in published maps and institutional affiliations.

(c) (i) Open Access This article is licensed under a Creative Commons Attribution 4.0 International License, which permits use, sharing, adaptation, distribution and reproduction in any medium or format, as long as you give appropriate credit to the original author(s) and the source, provide a link to the Creative Commons license, and indicate if changes were made. The images or other third party material in this article are included in the article's Creative Commons license, unless indicated otherwise in a credit line to the material. If material is not included in the article's Creative Commons license and your intended use is not permitted by statutory regulation or exceeds the permitted use, you will need to obtain permission directly from the copyrigh holder. To view a copy of this license, visit http://creativecommons.org/licenses/by/4.0/.

(c) The Author(s) 2021 